\author{
Ecología
}

\title{
Diversidad de macroinvertebrados fitófilos en arroyos de cabecera en pastizales de altura en Córdoba, Argentina
}

\author{
Phytophilous macroinvertebrate diversity in headwater streams in mountain grasslands in Córdoba, \\ Argentina \\ María Pía Rodríguez ${ }^{\mathrm{a}, \mathrm{b}}$, Romina E. Principe ${ }^{\mathrm{a}, \mathrm{c}, *}$, Javier A. Márquez ${ }^{\mathrm{a}, \mathrm{c}}$ y Graciela B. Raffaini ${ }^{\mathrm{c}}$ \\ ${ }^{a}$ Consejo Nacional de Investigaciones Científicas y Técnicas (CONICET), Argentina \\ ${ }^{\mathrm{b}}$ Departamento de Geología, Universidad Nacional de Río Cuarto, Ruta $36 \mathrm{Km} 601$ X5804BYA, Apartado postal Núm. 3, Río Cuarto, Córdoba, Argentina \\ ${ }^{\mathrm{c}}$ Departamento de Ciencias Naturales, Universidad Nacional de Río Cuarto, Ruta 36 Km 601 X5804BYA, Apartado postal Núm. 3, Río Cuarto, Córdoba, Argentina
}

Recibido el 26 de septiembre de 2016; aceptado el 12 de junio de 2017

Disponible en Internet el 6 de diciembre de 2017

\section{Resumen}

Las macrófitas cumplen un rol importante en los sistemas lóticos, incrementando la heterogeneidad del hábitat, proveyendo alimento y refugio para la fauna. El objetivo del estudio fue analizar la estructura y composición de la comunidad de macroinvertebrados asociados a macrófitas en arroyos de cabecera en pastizales de altura (subcuenca Ctalamochita, Córdoba, Argentina) en 2 períodos hidrológicos. Asimismo, se evaluó la estructura de los ensambles de macroinvertebrados presentes en diferentes especies de macrófitas (Hydrocotyle spp., Rorippa nasturtium-aquaticum y Ranunculus flagelliformis). Se muestrearon 3 arroyos durante los periodos hidrológicos de aguas altas (marzo de 2012) y aguas bajas (agosto de 2012). En cada período se colectaron muestras de macroinvertebrados asociados a la macrófita dominante en cada arroyo. Se identificaron 72 taxones de macroinvertebrados fitófilos. Los más frecuentes y abundantes fueron Naidinae, Hyallela curvispina, Americabaetis spp., Corynoneura spp. y Rheotanytarsus spp. La composición de los ensambles difirió entre períodos hidrológicos y se detectó una mayor abundancia de macroinvertebrados en $R$. nasturtium-acuaticum. Los resultados demuestran que los parches de macrófitas en los arroyos de cabecera en pastizales de altura albergan una fauna de macroinvertebrados abundante y diversa, y proporcionan a su vez importante información de base para el biomonitoreo de estos sistemas acuáticos.

(C) 2017 Universidad Nacional Autónoma de México, Instituto de Biología. Este es un artículo Open Access bajo la licencia CC BY-NC-ND (http://creativecommons.org/licenses/by-nc-nd/4.0/).

Palabras clave: Ecosistema lótico; Insectos acuáticos; Macrófitas; Hábitat; Hidroperiodo

\begin{abstract}
Macrophytes play an important role in lotic systems increasing habitat heterogeneity and providing food and shelter for wildlife. The aim of the study was to analyze the structure and composition of the invertebrate community associated with macrophytes in headwater streams in mountain grasslands (Ctalamochita sub-basin, Córdoba, Argentina) in two hydrological periods. The structure of the invertebrate assemblages in different macrophyte species (Hydrocotyle spp., Rorippa nasturtium-aquaticum and Ranunculus flagelliformis) was also evaluated. Three streams were sampled during high water (March 2012) and low water (August 2012) period. In each period, samples of invertebrates associated with the dominant macrophyte in each stream were collected. Seventy-two taxa of phytophilous macroinvertebrates were identified. The most frequent and abundant taxa were Naidinae, Hyalella curvispina, Americabaetis spp., Corynoneura spp. and Rheotanytarsus spp. Assemblages composition differed between hydrological periods and greater abundance of invertebrates was detected in $R$. nasturtium-aquaticum. The results showed that macrophyte patches in grassland headwater streams harbor an abundant and diverse invertebrate fauna and provide important base information for biomonitoring.
\end{abstract}

(C) 2017 Universidad Nacional Autónoma de México, Instituto de Biología. This is an open access article under the CC BY-NC-ND license (http://creativecommons.org/licenses/by-nc-nd/4.0/).

Keywords: Lotic ecosystem; Aquatic insects; Macrophytes; Habitat; Hydroperiod

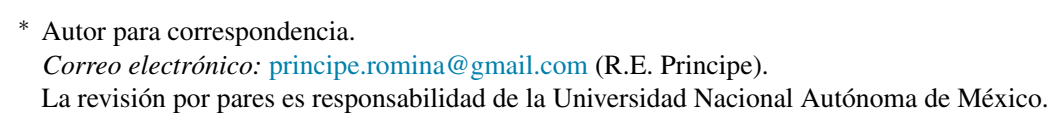




\section{Introducción}

Las plantas acuáticas son de suma importancia en la configuración del ambiente fluvial y cumplen un rol preponderante en la estructura y funcionamiento de estos sistemas (Feijoó y Menéndez, 2009), ya que incrementan la complejidad y heterogeneidad de los hábitats acuáticos (Thomaz y Cunha, 2010). Estas plantas colonizan generalmente áreas poco profundas y modifican las características hidráulicas del cauce, reduciendo la velocidad de la corriente y favoreciendo la retención del sedimento y de la materia orgánica (Argentina, Freeman y Freeman, 2010; SandJensen y Mebus, 1996). De este modo las macrófitas modifican la estructura del hábitat y los recursos alimentarios disponibles influyendo sobre la fauna acuática (Humphries, 1996; Thomaz y Cunha, 2010).

Las macrófitas albergan una gran variedad de macroinvertebrados (macroinvertebrados fitófilos sensu Allan y Castillo, 2007; Habib y Yousub, 2015) los cuales pueden utilizar estas plantas como alimento, refugio, sitios de oviposición y como sustrato (Habib y Yousub, 2015). La densidad y diversidad de macroinvertebrados son más elevadas en las zonas cubiertas por macrófitas (Rozas y Odum, 1988; Uvíra, Uvírová y Komárek, 2005) y muchas especies viven exclusivamente asociadas a determinadas macrófitas debido a que alguna etapa de su ciclo vital depende de la planta (Feijoó y Menéndez, 2009; Gopal y Goel, 1993). Asimismo, se ha reportado que la diversidad de macroinvertebrados fitófilos es mayor en macrófitas de arquitectura más compleja (Abendroth, Ramsay, Foggo, Rundle y Bilton, 2005; Taniguchi, Nakano y Tokeshi, 2003; Thomaz, Dibble, Evangelista, Higuti y Bini, 2008; Warfe y Barmuta, 2006). De esta forma las macrófitas aportan un componente estructural a los arroyos que incrementa la diversidad de especies (Thomaz y Cunha, 2010).

Las nacientes de los arroyos de las Sierras de Córdoba drenan pastizales de altura, por lo que en estos arroyos no existe sombreado sobre el lecho, y la fuente de carbono orgánico proviene principalmente de la fotosíntesis de la comunidad algal (Thompson y Townsend, 2004; Whiting, Whiles y Stone, 2011). Si bien el sustrato predominante son rocas y bloques, ya que las nacientes se ubican en una región montañosa, las macrófitas crecen en áreas de deposición donde se acumula el sedimento más fino, conformando parches de vegetación acuática que cubren normalmente un $10 \%$ de un tramo y en algunos casos pueden presentar hasta un $70 \%$ de cobertura. La fauna de macroinvertebrados fitófilos ha sido estudiada principalmente en arroyos de llanura (Damborsky, Poi y Mazza, 2012; Feldman, 2001; Ferreiro, Feijoó, Giorgi y Leggieri, 2011; Poi de Neiff y Neiff, 2006; Tarjányi y Berczik, 2014) siendo escasa la información sobre estos ensambles en arroyos de cabecera de regiones montañosas. Los objetivos del presente estudio son: 1) analizar la estructura y composición de los ensambles de macroinvertebrados asociados a macrófitas en arroyos de cabecera que drenan pastizales de altura; 2) comparar los ensambles de macroinvertebrados fitófilos entre periodos de alto y bajo caudal; y 3) evaluar su estructura en diferentes especies de macrófitas.

\section{Materiales y métodos}

El área de estudio comprendió arroyos de la subcuenca superior del río Ctalamochita (Tercero). En esta zona los arroyos nacen entre los 1,600 y los $900 \mathrm{~m}$ snm en la falda oriental de las sierras Grandes de la provincia de Córdoba, Argentina (DíazLozada et al., 2015). Los ríos y arroyos del área discurren sobre lechos rocosos de fuertes pendientes (12-45\%), lo que caracteriza la morfología de estos cursos angostos y encajonados, de comportamiento erosivo y régimen turbulento (Cabido et al., 2003). El periodo lluvioso ocurre entre la primavera y el verano (octubre-marzo) con un promedio de precipitación anual de $725 \mathrm{~mm}$. La temperatura máxima alcanza los $34^{\circ} \mathrm{C}$ en el verano (diciembre-marzo) y en el invierno (junio-septiembre) se registra una mínima de $-5^{\circ} \mathrm{C}$ (Cabido et al., 2003). La vegetación del área de estudio responde a un gradiente altitudinal formando pisos o zonas de vida (Cabido et al., 2003). Los arroyos estudiados se ubican en el piso altitudinal entre 1,100 y $1,700 \mathrm{~m} \mathrm{snm}$, dominado por pastizales con predominio de algunas especies de los géneros Festuca y Stipa. Para este estudio se seleccionaron 3 arroyos de primer orden (arroyo $1: 31^{\circ} 58^{\prime} 56^{\prime \prime} \mathrm{S}$, $64^{\circ} 43^{\prime} 25^{\prime \prime} \mathrm{O}$; arroyo 2: $31^{\circ} 58^{\prime} 52^{\prime \prime} \mathrm{S}$, $64^{\circ} 46^{\prime} 29^{\prime \prime} \mathrm{O}$; arroyo 3: $\left.31^{\circ} 58^{\prime} 47^{\prime \prime} \mathrm{S}, 64^{\circ} 48^{\prime} 41^{\prime \prime} \mathrm{O}\right)$ en sitios de semejante exposición, altitud (arroyo 1: 1,130 $\mathrm{m} \mathrm{snm}$, arroyo 2: 1,157 $\mathrm{m} \mathrm{snm}$, arroyo 3: $1,175 \mathrm{~m} \mathrm{snm}$ ) y superficie de drenaje (arroyo 1: 31 ha, arroyo 2: $88 \mathrm{ha}$, arroyo 3: $87 \mathrm{ha}$ ) pertenecientes a la subcuenca del arroyo Santa Rosa. Estos 3 arroyos corresponden a microcuencas naturales de pastizales de altura sujetos a una baja presión de pastoreo $\mathrm{y}$ a incendios eventuales.

Se recolectaron muestras de macroinvertebrados asociados a macrófitas en cada arroyo durante el periodo hidrológico de aguas altas (marzo de 2012) y el periodo de aguas bajas (agosto de 2012). En un tramo de $20 \mathrm{~m}$ se determinó visualmente la cobertura proporcional de macrófitas. Sobre diferentes parches de la especie de macrófita dominante en cada arroyo y período se obtuvieron 3 muestras de macroinvertebrados fitófilos con un copo entomológico de volumen conocido (diámetro: $32 \mathrm{~cm}$, malla: $300 \mu \mathrm{m}$ ). Las muestras se fijaron en formol $4 \%$ en el campo y se conservaron en alcohol $70 \%$ luego de su procesamiento en el laboratorio. Durante la toma de muestras se registraron variables físico-químicas para la caracterización de los arroyos utilizando sensores portátiles: profundidad y velocidad de corriente (Global Flow Probe FP101) y conductividad, $\mathrm{pH}$ y temperatura del agua (Multiparameter PCSTestr 35, EutechOakton). Para una adecuada caracterización de los arroyos, la profundidad y la velocidad de corriente se midieron en los 2 hábitats fluviales predominantes de los arroyos: pozas y rabiones, los cuales se distinguen marcadamente en estas variables.

Los macroinvertebrados recolectados fueron cuantificados e identificados en el laboratorio con claves taxonómicas especializadas (Domínguez y Fernández, 2009). Los datos de densidad de las muestras fueron expresados en número de individuos (ind.) por $\mathrm{m}^{3}, \mathrm{~m}^{2}$ y por peso seco de macrófita para considerar la variación potencial de la densidad debida a la complejidad estructural diferencial de las distintas especies de plantas. La superficie ocupada por las macrófitas incluidas en cada muestra se estimó en 
el laboratorio extendiendo los ejemplares de manera que ocupasen completamente un rectángulo. Adicionalmente, las plantas se secaron en estufa a $60^{\circ} \mathrm{C}$ durante $48 \mathrm{~h}$ para obtener su peso seco.

\section{Análisis de los datos}

Se graficaron curvas de rango abundancia (Magurran, 1988) para evaluar los cambios en la composición taxonómica entre periodos hidrológicos considerando solo los taxones con una abundancia relativa mayor o igual al 1\%. Se realizó además un escalamiento no métrico multidimensional (NMDS) como método de ordenación utilizando el coeficiente de similitud de Bray-Curtis en el programa PAST versión 3.13 (Hammer, Harper y Ryan, 2001). Para la confección de las curvas y para el NMDS se utilizaron los datos de abundancia expresados en $\mathrm{m}^{2}$. La significación estadística de los grupos obtenidos por el NMDS fue comprobada estadísticamente mediante un análisis de similitudes (Anosim).

La caracterización estructural de los ensambles se determinó mediante el cálculo de la abundancia total y de los índices de diversidad de Shannon, Simpson y Alfa, y de equitatividad de Shannon y de dominancia de Simpson (Magurran, 1988). Se realizaron pruebas de Kruskal-Wallis para comparar los atributos estructurales de los ensambles de macroinvertebrados entre las diferentes especies de macrófitas utilizando el programa INFOSTAT (Di Rienzo et al., 2012). Los análisis se realizaron para todos los índices de diversidad y equitatividad y para las 3 medidas de abundancia de macroinvertebrados (ind.$/ \mathrm{m}^{3}$, ind. $/ \mathrm{m}^{2}$ e ind. $/ \mathrm{g}$ de peso seco de macrófita).

\section{Resultados}

Los datos sobre las macrófitas dominantes en cada arroyo y período hidrológico se resumen en la tabla 1. La cobertura del lecho varió entre los arroyos, destacándose el arroyo 1 con un porcentaje de cobertura de macrófitas marcadamente mayor (70\%) que los arroyos 2 y 3 (entre $1 \%$ y $10 \%$ de cobertura). La velocidad de corriente media de los tramos estudiados fue de $0.46 \mathrm{~m} / \mathrm{s}$ en aguas altas y de $0.27 \mathrm{~m} / \mathrm{s}$ en aguas bajas, mientras que la profundidad media varió entre $0.20 \mathrm{~m}$ y $0.12 \mathrm{~m}$ en aguas altas y bajas respectivamente. La temperatura media del agua durante el periodo de alto caudal fue de $18.8^{\circ} \mathrm{C}$ y en bajo caudal fue de $15^{\circ} \mathrm{C}$. La conductividad osciló entre $14 \mu \mathrm{S} / \mathrm{cm}$ y $179 \mu \mathrm{S} / \mathrm{cm}$ y el rango de $\mathrm{pH}$ estuvo entre 7.6 y 9.6.

Se identificaron 72 taxones de macroinvertebrados fitófilos (tabla 2). Los más frecuentes y abundantes fueron Naidinae, Hyallela curvispina Shoemaker, 1942, Americabaetis spp., Corynoneura spp. y Rheotanytarsus spp. Las curvas de rango abundancia mostraron una diferenciación estacional en la composición taxonómica de los ensambles (fig. 1). Durante el período de aguas bajas Naidinae fue dominante, mientras que en el período de aguas altas dominó junto con Corynoneura spp. En el periodo de aguas bajas se observó una disminución marcada de la abundancia de Parametriocnemus spp., Austrelmis spp., Pseudochironomus spp. y Dugesidae, y un incremento en la densidad de Americabaetis spp., Rheotanytarsus spp. y Cricotopus spp.

El NMDS mostró diferencia en los ensambles de macroinvertebrados fitófilos, principalmente entre los períodos hidrológicos (fig. 2), la cual fue significativa de acuerdo con el resultado del Anosim $(R=0.3093 ; p=0.0015)$. Asimismo, los ensambles asociados a diferentes especies de macrófitas no presentaron diferencias (Anosim; $\mathrm{R}=0.1139 ; \mathrm{p}=0.1403$ ). De los atributos estructurales que fueron analizados en los ensambles estudiados solamente la abundancia total de macroinvertebrados cuantificada en $\mathrm{m}^{3}$ exhibió diferencias entre las especies de macrófitas (pruebas de Kruskal-Wallis [tabla 3]). Esta variable resultó significativamente mayor en la macrófita $R$. nasturtium-aquaticum.

\section{Discusión}

Los parches de macrófitas muestreados en este estudio albergaron una diversa fauna de macroinvertebrados acuáticos pertenecientes a 72 taxones, dentro de la cual predominaron Naidinae, Hyallela curvispina, Americabaetis spp., Corynoneura spp. y Rheotanytarsus spp. Otros autores reportaron para arroyos de llanura un número considerablemente menor de taxones totales, entre 9 y 27 (Arimoro, Ogbeibu y Raifu, 2007; Damborsky et al., 2012; Ferreiro et al., 2011), a pesar de que en estos arroyos la vegetación acuática forma densos parches que cubren gran parte del lecho. De esta manera, nuestro estudio resalta la importancia de los parches de macrófitas en arroyos de montaña como hábitat de una rica fauna de macroinvertebrados.

En los arroyos estudiados numerosos taxones de dípteros de la familia Chironomidae presentaron elevada abundancia en los parches de vegetación acuática. Si bien estos organismos habitan en una gran variedad de ambientes, por lo general prefieren lugares de remansos y protegidos de las fuertes corrientes (Paggi, 2009), tales como los que ofrecen las plantas acuáticas. H. curvispina y Americabaetis spp. resultaron también muy abundantes en las macrófitas relevadas, lo cual concuerda con lo reportado por otras investigaciones donde se registraron elevadas abundancias de anfípodos del género Hyallella spp. en plantas acuáticas (Casset, Momo y Giorgi, 2001; Giorgi, Poncio, Dutweiler, Martinelli y Feijoó, 1996; Saigo, Marchese y Montalto, 2009) atribuyendo la presencia de estos macroinvertebrados principalmente al refugio que la vegetación provee (Casset et al., 2001). Asimismo, dentro de los géneros de la familia Baetidae, Americabaetis spp. presenta una marcada afi-

Tabla 1

Especies de macrófitas muestreadas en cada arroyo y periodo hidrológico de la subcuenca del arroyo Santa Rosa (Córdoba, Argentina).

\begin{tabular}{|c|c|c|c|}
\hline & Arroyo 1 & Arroyo 2 & Arroyo 3 \\
\hline Aguas altas & Hydrocotyle spp. & Ranunculus flageliformis Hydrocotyle spp. & Hydrocotyle spp. \\
\hline Aguas bajas & Rorippa nasturtium-aquaticum & Ranunculus flageliformis & Hydrocotyle spp. Rorippa nasturtium-aquaticum \\
\hline
\end{tabular}


Tabla 2

Lista de macroinvertebrados acuáticos colectados en parches de macrófitas en arroyos de cabecera en pastizales de altura. Se presenta la frecuencia relativa (\% F) y la abundancia total de cada taxón.

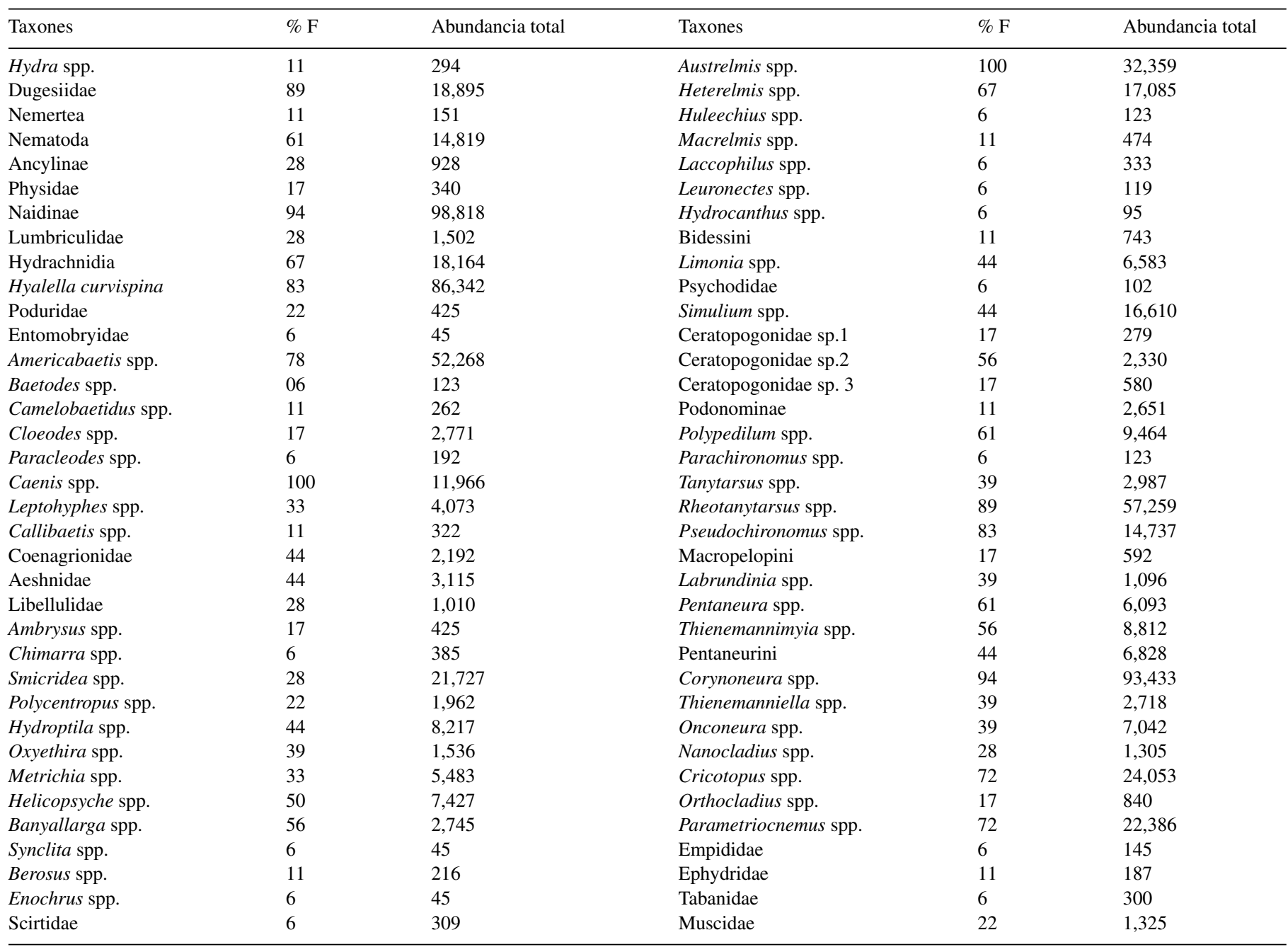

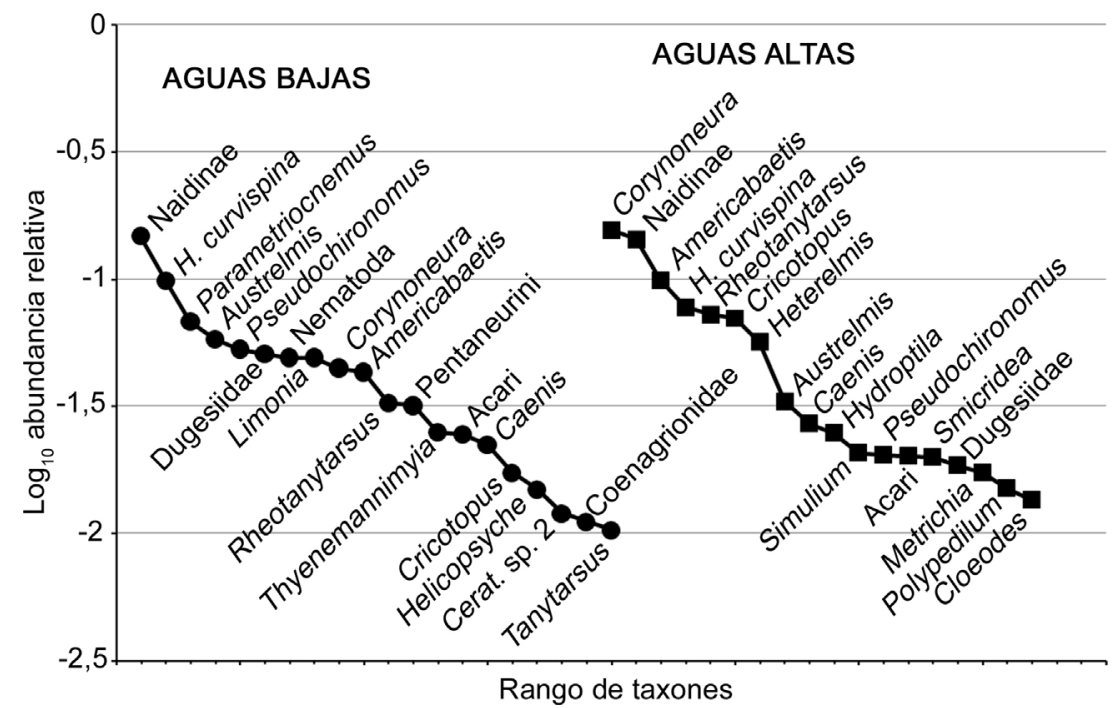

Figura 1. Curvas de rango abundancia del ensamble de macroinvertebrados fitófilos en arroyos de cabecera en pastizales de altura durante los períodos de aguas altas y aguas bajas. 


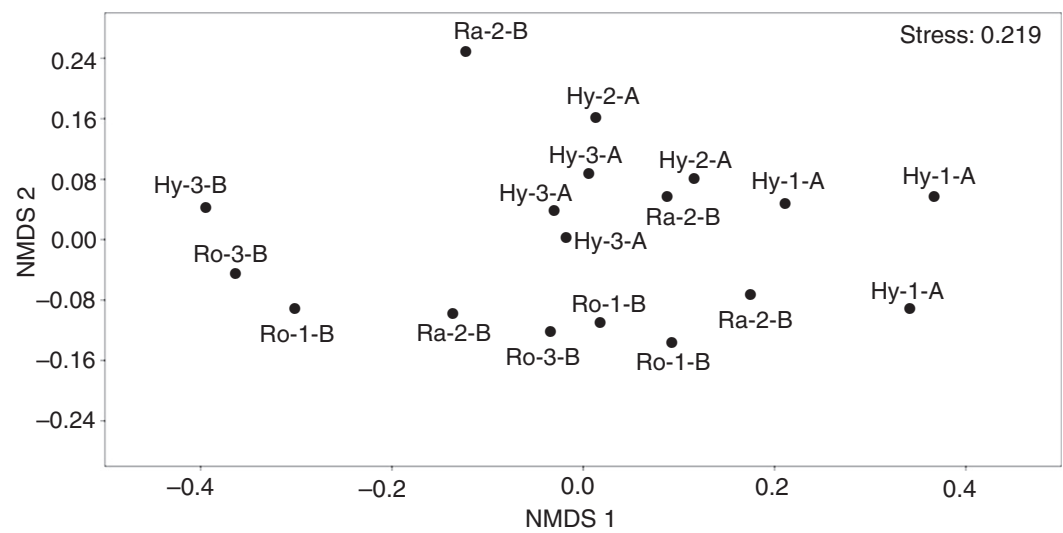

Figura 2. NMDS de muestras de macroinvertebrados recolectados en parches de macrófitas (Hy: Hydrocotyle spp.; Ra: Ranunculus flagelliformis; Ro: Rorippa nasturtium-aquaticum) de 3 arroyos de cabecera en pastizales de altura (1, 2 y 3 ) durante los periodos de aguas altas (A) y aguas bajas (B).

nidad por la vegetación acuática (Domínguez, Molineri y Nieto, 2009). Los oligoquetos de la subfamilia Naidinae dominaron el ensamble de macroinvertebrados fitófilos en ambos periodos hidrológicos. Estos macroinvertebrados pueden encontrarse en una gran variedad de hábitats acuáticos, con preferencia por los sedimentos finos, donde suelen presentar mayor densidad (Marchese, 2009). En este sentido, los parches de macrófitas actúan como un filtro de sedimentos finos (Argentina et al., 2010; Sand-Jensen y Mebus, 1996), los cuales se acumulan en los parches propiciando la colonización por oligoquetos de agua dulce.

Los resultados del estudio mostraron diferencias estacionales en la composición taxonómica de los ensambles de macroinvertebrados fitófilos, lo cual puede estar asociado a las variaciones periódicas en la morfología de las plantas, que alternan morfotipos contrastantes en época de crecimiento y senescencia (Lillie y Budd, 1992). Alternativamente, en épocas de alto caudal los eventos de crecidas pueden provocar la deriva de macroinvertebrados que encuentren sitios de anclaje en la vegetación acuática (Pardo y Armitage, 1997).

La comparación de los índices estructurales de la comunidad de macroinvertebrados entre parches de diferentes especies de macrófitas (Hydrocotyle spp., $R$. flageliformis, $R$. nasturtiumaquaticum) solamente exhibió diferencias en la abundancia de macroinvertebrados cuantificada en $\mathrm{m}^{3}$. Rorippa nasturtiumaquaticum fue la especie que albergó el mayor número de individuos, lo cual puede deberse a la mayor densidad de follaje que presenta esta planta. Ferreiro et al. (2011) y Walker, Wijnhoven y van der Velde (2012) estudiaron los ensambles de macroinvertebrados que albergan macrófitas con diferente complejidad arquitectónica, encontrando que especies de arquitectura más compleja sustentan mayor abundancia de macroinvertebrados, pero no necesariamente mayor riqueza y diversidad. Sin embargo, las especies de macrófitas relevadas en nuestro estudio no estuvieron representadas igualitariamente entre los diferentes arroyos y periodos hidrológicos, por lo que este resultado constituye solamente una primera aproximación sobre posibles diferencias entre los ensambles de macroinvertebrados que habitan diferentes especies de macrófitas en estos arroyos de cabecera.
Tabla 3

Resultados de las pruebas de Kruskal-Wallis aplicadas para comparar los atributos estructurales de los ensambles de macroinvertebrados fitófilos entre diferentes especies de macrófitas: Hydrocotyle spp., Rorippa nasturtiumaquaticum y Ranunculus flagelliformis.

\begin{tabular}{lll}
\hline Variable & $\mathrm{H}$ & $p$ \\
\hline Abundancia (ind./g) & 2.43 & 0.2965 \\
Abundancia (ind./m ${ }^{2}$ ) & 1.44 & 0.4875 \\
Abundancia (ind./m ${ }^{3}$ ) & $\mathbf{6 . 3 6}$ & $\mathbf{0 . 0 4 1 6}$ \\
Riqueza & 0.52 & 0.7700 \\
Alfa & 1.25 & 0.5349 \\
Shannon H' & 0.89 & 0.6403 \\
Simpson 1/D & 0.70 & 0.7033 \\
Shannon J' & 1.30 & 0.5226 \\
Simpson D & 0.70 & 0.7033 \\
\hline
\end{tabular}

Los valores resaltados en negrita corresponden a los valores de p estadísticamente significativos de acuerdo al estadístico $\mathrm{H}$ de las pruebas.

El estudio de los ensambles de macroinvertebrados fitófilos en cuencas de altura es de particular importancia, ya que brinda valiosa información sobre el uso del hábitat en estos arroyos, proveyendo la base para predecir respuestas bióticas frente a perturbaciones (Hawkins et al., 1993). En el caso de las sierras de Córdoba en la región central de Argentina los pastizales de altura han sido sometidos a diferentes acciones antrópicas, entre las que se encuentra la forestación comercial con especies arbóreas de crecimiento rápido, principalmente pinos (Jobbágy et al., 2006; Plevich, Núñez, Cantero, Demaestri y Viale, 2002). Esta modificación en el uso de la tierra se extiende hasta las riberas de los arroyos, provocando alteraciones en la intensidad lumínica que incide sobre los mismos (Principe, Márquez, Cibils-Martina, Jobbágy y Albariño, 2015). Esta situación plantea potenciales efectos sobre la diversidad y abundancia de plantas acuáticas, y de manera concomitante sobre la fauna de macroinvertebrados fitófilos. El presente trabajo resalta la importancia de la vegetación acuática como un elemento estructural clave en los arroyos de altura, aportando además valiosa información de base a ser utilizada en el monitoreo de la biodiversidad. Futuros estudios que analicen la partición del hábitat en arroyos serranos de cabecera permitirán confirmar si las macrófitas actúan como hábitats 
exclusivos y en qué magnitud incrementan el elenco de especies a escala de tramo.

\section{Agradecimientos}

Los autores agradecemos a L. Cibils-Martina por su asistencia en las tareas de campo. Deseamos agradecer también las sugerencias y comentarios de 2 revisores anónimos para mejorar el manuscrito. El presente estudio fue realizado con financiamiento de la Agencia Nacional de Promoción Científica y Tecnológica, Argentina (ANPCYT, PICT 1113) y del Ministerio de Ciencia y Tecnología del Gobierno de la Provincia de Córdoba (MCYT, GRF 2008).

\section{Referencias}

Allan, J. D. y Castillo, M. M. (2007). Stream ecology: structure and function of running waters (2nd ed.). Dordrecht, The Netherlands: Springer

Argentina, J. E., Freeman, M. C. y Freeman, B. J. (2010). The response of stream fish to local and reach-scale variation in the occurrence of a benthic aquatic macrophyte. Freshwater Biology, 55, 643-653.

Arimoro, F. O., Ogbeibu, A. E. y Raifu, F. F. (2007). Phytophilous macroinvertebrates of floating Nymphaea lotus and Pistia stratiotes in River Orogodo, Niger Delta, Nigeria. Tropical Freshwater Biology, 16, 75-87.

Cabido, D., Cabido, M., Garre, S. M., Gorgas, J. A., Miatello, R., Rambaldi, S., et al. (2003). Regiones naturales de la provincia de Córdoba. Serie C. Córdoba, Argentina: Publicaciones técnicas. Agencia Córdoba. Dirección de ambiente.

Casset, M. A., Momo, F. R. y Giorgi, A. D. N. (2001). Dinámica poblacional de dos especies de anfípodos y su relación con la vegetación acuática en un microambiente de la cuenca del río Luján (Argentina). Ecología Austral, 11, 79-85.

Damborsky, M. P., Poi, A. S. G. y Mazza, S. (2012). Patrón espacial y temporal de las colectividades de artrópodos asociados a macrófita en un río subtropical de bajo orden (Chaco, Argentina). Interciencia, 37, 534-541.

Di Rienzo, J. A., Casanoves, F., Balzarini, M. G., González, L., Tablada, M. y Robledo, C. W. (2012). InfoStat versión 2012. Grupo InfoStat, FCA: Universidad Nacional de Córdoba, Argentina. Disponible en: http://www.infostat.com.ar.

Díaz-Lozada, J. M., García, C. M., Herrero, H., Barchiesi, G. M., Romagnoli, M., Portapila, M., et al. (2015). Cuantificación del escurrimiento superficial de la cuenca del río Carcarañá. Revista de la Facultad de Ciencias Exactas, Físicas y Naturales, 2, 59-72.

Domínguez, E. y Fernández, H. R. (2009). Macroinvertebrados bentónicos sudamericanos: sistemática y biología. Tucumán, Argentina: Fundación M. Lillo.

Domínguez, E., Molineri, C. y Nieto, C. (2009). Ephemeroptera. En E. Domínguez y H. Fernández (Eds.), Macroinvertebrados bentónicos sudamericanos: sistemática y biología (pp. 551-565). Tucumán, Argentina: Fundación Miguel Lillo.

Feijoó, C. y Menéndez, M. (2009). La biota de los ríos: los macrófitos. En A. Elosegi y S. Sabater (Eds.), Conceptos y técnicas en ecología fluvial (pp. 243-251). Madrid: Fundación BBVA.

Feldman, R. S. (2001). Taxonomic and size structures of phytophilous macroinvertebrate communities in Vallisneria and Trapa beds of the Hudson River, New York. Hydrobiologia, 452, 233-245.

Ferreiro, N., Feijoó, C., Giorgi, A. y Leggieri, L. (2011). Effects of macrophyte heterogeneity and food availability on structural parameters of the macroinvertebrate community in a Pampean stream. Hydrobiologia, 664, 199-211.

Giorgi, A. D. N., Poncio, G., Dutweiler, F., Martinelli, F. y Feijoó, C. (1996). Variación estacional de la abundancia de moluscos y anfípodos en un arroyo de llanura. VI Jornadas Pampeanas de Ciencias Naturales, 1, 36-37.

Gopal, B. y Goel, U. (1993). Competition and allelopathy in aquatic plant communities. The Botanical Review, 59, 155-210.
Habib, S. y Yousuf, A. R. (2015). Effect of macrophytes on phytophilous macroinvertebrate community: A review. Journal of Entomology and Zoology Studies, 3, 377-384.

Hammer, Ø., Harper, D. A. T. y Ryan, P. D. (2001). Paleontological statistics software: package for education and data analysis. Palaeontología Electronica, $4,1-9$.

Hawkins, C. P., Kershner, J. L., Bisson, P. A., Bryant, M. D., Decker, L. M., Gregory, S. V., et al. (1993). A hierarchical approach to classifying stream habitat features. Fisheries, 18, 3-12.

Humphries, P. (1996). Aquatic macrophytes, macroinvertebrate associations and water levels in a lowland Tasmanian river. Hydrobiologia, 321, 219-233.

Jobbágy, E. G., Vasallo, M., Farley, K. A., Piñeiro, G., Garbulsky, M. F., Nosetto, M. D., et al. (2006). Forestación en pastizales: hacia una visión integral de sus oportunidades y costos ecológicos. Agrociencia, 10, 109-124.

Lillie, R. A. y Budd, J. (1992). Habitat architecture of Myriophyllum spicatum L. as an index to habitat quality for fish and macroinvertebrates. Freshwater Ecology, 7, 113-125.

Mac Abendroth, L., Ramsay, P. M., Foggo, A., Rundle, S. D. y Bilton, D. T. (2005). Does macrophyte fractal complexity drive invertebrate diversity, biomass and body size distributions? Oikos, 111, 279-290.

Magurran, A. E. (1988). Ecological diversity and its measurement. London: Croom Helm.

Marchese, M. (2009). Annelida Oligochaeta. En E. Domínguez y H. Fernández (Eds.), Macroinvertebrados bentónicos sudamericanos: sistemática y biología (pp. 551-565). Tucumán, Argentina: Fundación Miguel Lillo.

Paggi, A. C. (2009). Diptera, Chironomidae. En E. Domínguez y H. Fernández (Eds.), Macroinvertebrados bentónicos sudamericanos: sistemática y biología (pp. 383-409). Tucumán, Argentina: Fundación Miguel Lillo.

Pardo, I. y Armitage, P. D. (1997). Species assemblages as descriptors of mesohabitats. Hydrobiologia, 344, 111-128.

Plevich, J., Núñez, C., Cantero, J., Demaestri, M. y Viale, S. (2002). Biomasa del pastizal bajo diferentes densidades de pino (Pinus elliottii). Agroforestería de las Américas, 9, 19-23.

Poi de Neiff, A. S. G. y Neiff, J. J. (2006). Riqueza de especies y similaridad de los invertebrados que viven en plantas flotantes de la planicie de inundación del río Paraná (Argentina). Interciencia, 31, 220-225.

Principe, R. E., Márquez, J. A., Cibils-Martina, L., Jobbágy, E. G. y Albariño, R. J. (2015). Pine afforestation changes more strongly community structure than ecosystem functioning in grassland mountain streams. Ecological Indicators, 57, 366-375.

Rozas, L. P. y Odum, W. E. (1988). Occupation of submerged aquatic vegetation by fishes- testing the roles of food and refuge. Oecología, 77, 101-106.

Saigo, M., Marchese, M. y Montalto, L. (2009). Hábitos alimentarios de Hyalella curvispina Shoemaker, 1942 (Amphipoda: Gammaridea) en ambientes leníticos de la llanura alluvial del río Paraná Medio. Natura Neotropicalis, $40,43-59$.

Sand-Jensen, K. y Mebus, J. R. (1996). Fine scale patterns of water velocity within macrophyte patches in streams. Oikos, 76, 169-180.

Taniguchi, H., Nakano, S. y Tokeshi, M. (2003). Influences of habitat complexity on the diversity and abundance of epiphytic invertebrates on plants. Freshwater Biology, 48, 718-728.

Tarjányi, N. y Berczik, A. (2014). Spatial distribution of phytophilous macroinvertebrates in a side arm of the middle Danube River. Acta Zoologica Bulgarica, 7, 13-17

Thomaz, S. M. y Cunha, E. R. D. (2010). The role of macrophytes in habitat structuring in aquatic ecosystems: methods of measurement, causes and consequences on animal assemblages composition and biodiversity. Acta Limnologica Brasiliensia, 22, 218-236.

Thomaz, S. M., Dibble, E. D., Evangelista, L. R., Higuti, J. y Bini, L. M (2008). Influence of aquatic macrophyte habitat complexity on invertebrate abundance and richness in tropical lagoons. Freshwater Biology, 53, 358-367.

Thompson, R. M. y Townsend, C. R. (2004). Land-use influences on New Zealand stream communities: effects on species composition, functional organization, and food-web structure. New Zealand Journal of Marine and Freshwater Research, 38, 595-608.

Uvíra, V., Uvírová, I. y Komárek, O. (2005). Comparison of macrozoobenthic community structure within vegetative and mineral substrata 
in a stream rich in submerged macrophytes. Verhandlungen Internationale Vereinigungfúr Theoresticheund Angewandte Limnologe, 29, 1054-1058.

Walker, P. D., Wijnhoven, S. y van der Velde, G. (2012). Macrophyte presence and growth form influence macroinvertebrate community structure. Aquatic Botany, 104, 80-87.
Warfe, D. M. y Barmuta, L. A. (2006). Habitat structural complexity mediates food web dynamics in a freshwater macrophyte community. Oecologia, 150, $141-154$.

Whiting, D. P., Whiles, M. R. y Stone, M. L. (2011). Patterns of macroinvertebrate production, trophic structure, and energy flow along a tallgrass prairie stream continuum. Limnology and Oceanography, 56, 887-898. 